\title{
Die intravenöse Fettzufuhr als Fettbelastungstest
}

\author{
Von \\ H. SchöN, W. ZeLLER, U. KRAUSE und N. HenNing \\ Aus der Medizinischen Klinik der Universität Erlangen-Nürnberg (Direktor: Prof. Dr. N. Henning)
}

(Der Schriftleitung zugegangen am 3. Oktober 1962)

\begin{abstract}
Im Gegensatz zu Ergebnissen anderer Untersucher läßt sich durch intravenöse Verabreichung emulgierten Fettes bei Patienten mit einem Grundleiden auf atherosklerotischer Basis eine Verlangsamung des Fettabtransportes im Vergleich zu Gesunden nachweisen. Die Ursachen dieser Diskrepanz werden diskutiert. Bei Langzeittherapie mit nicht überdosierter intravenöser Fettzufuhr wurde bei einigen Patienten eine Retentionshyperlipämie beobachtet, die als Hypertriglyzeridämie angesprochen werden muß. Die intravenöse Fettzufuhr ist ein brauchbarer und exakt dosierbarer Fettbelastungstest zur Erkennung von Störungen des Fettabtransportes aus dem Blut.
\end{abstract}

Contrary to the findings of other authors, a retarded rate of fat removal can be demonstrated in atheroslerotic patients, compared with healthy individuals, by the intravenous administration of emulsified fat. The reasons for this contradiction are discussed. In long term therapy with intravenous sub-doses of fat, some patients showed a retention hyperlipaemia, which must be a hypertriglycerideaemia. Intravenous injection of fat permits exact dosing and is a useful fat tolerance test for the detection of disturbances in the normal removal of fat from the blood.

Von verschiedenen Autoren ist darauf hingewiesen worden, daß bei Patienten. mit Koronarsklerose die Hyperlipämie nach Zufuhr von üblichen Nahrungsfetten oder markiertem Triolein länger anhält als bei gefäßgesunden Personen (1-4). Insbesondere konnte ThanNhauser (5) bereits 1949 durch Verfütterung von $\mathrm{J}^{131}$-markiertem Triolein den Nachweis erbringen, da $B$ bei der essentiellen Hyperlipämie das markierte Fett länger im Blut persistiert als bei normolipämischen Vergleichspersonen. Wenn auch die Verwendung markierten Fettes eine sehr viel exaktere Bestimmung der Plasmaverweildauer des resorbierten Nahrungsfettes zuläßt als die Belastung mit einem inaktiven Fett, so stand zu erwarten, daB mit der Fettzufuhr auf intravenösem Wege eine Reihe von Fehlermöglichkeiten, die der oralen Fettbelastung anhaften, umgangen werden könnten. Diese Überlegungen dürften MASHFORD und Mitarbeiter (6) sowie BouchIER und Mitarbeiter (7) veranlaßt haben, das Ausmaß und die Dauer der Hyperlipämie nach Infusion bei Patienten mit Koronarsklerose und Kontrollpersonen zu prüfen. Eigentümlicherweise zeigten diese Untersuchungen keinen Unterschied zwischen beiden Gruppen. Wir haben bereits andernorts kurz auf unsere Bedenken gegen die Yersuchsanordnung hingewiesen (8). In eigenen Untersuchuingen über die Häufigkeit einer Retentionshyperlipämie (von uns als Klärinsuffizienz (9) bezeichnet) bei Patienten mit Atherosklerose der verschiedenen Lokalisationsformen und bei solchen Erkrankungen, die erfahrungsgemä $\beta$ zu einer Atherosklerose prädisponieren, hatte sich gezeigt, daß bei 570 Patienten mit essentieller Hypertonie, Koronarsklerose, Myokardinfarkt, Diabetes mellitus und Fettsucht in $60-80 \%$ der Fälle ein pathologischer Fettabtransport aus dem Blut nachgewiesen werden kann (10). Bei einem Teil solcher Patienten wurde die Geschwindigkeit des Fettabtransportes nach Verabfolgung. von $500 \mathrm{ml}$ einer 10\%igen Fettemulsion untersucht. Im Gegensatz zu früheren Untersuchungen $(6,7)$ enthält diese Fettemulsion lediglich 1,2\% Sojaphosphatide und keinen Zusatz eines synthetischen Emulgators.

\section{Versuche}

Bei 26 Versuchspersonen wurde nach mindestens 12 stündigem Fasten eine Infusion von $500 \mathrm{ml}$ einer 10\%igen Fettemulsion ${ }^{1}$ ) innerhalb von 3 Stunden ausgeführt. Die Blutfettkonzentration wurde vor und nach der Infusion sowie 2 und 4 Stunden post infusionem untersucht. Während dieser Zeit wurde fettfreie Kost gereicht. Die Gesamt-Esterfettsäuren wurden nach der Methode von GEY und SCHÖN (11), die Phosphatide nach feuchter Veraschung nach LoHMANN und Mitarbeitern (12) und das Gesamt-Cholesterin nach ZLATKIS und Mitarbeitern (13) bestimmt. Die Versuchspersonen gliederten sich in folgende 3 Gruppen:

Gruppe I: 10 gesunde Vergleichspersonen; Gruppe II: $10 \mathrm{~Pa}-$ tienten mit atherosklerotischen Grundleiden und Klärinsuffizienz; Gruppe III: 6 Patienten nach mehrfacher intravenöser Fettzufuhr mit Inanition infolge des Grundleidens.

Zur genauen Kenntnis der Zusammensetzung der Fettemulsion wurden Phosphatidemulgator ${ }^{2}$ ) und Baumwollsaatöl ${ }^{3}$ ) der fettchemischen Analyse unterworfen. Die Ergebnisse zeigen die Tabellen 1 und 2.

\section{Sojaphosphatide}

Es wurden-dünnschichtchromatographisch folgende Komponenten nachgewiesen (Methodik siehe WAGNER (14)):

Glyzeride, Lezithin, Kephalin, Lysoverbindungen von Lezithin und Kephalin.

1) „Lipofundin“, (B. Braun, Melsungen).

2,8) Wir danken der Firma B. Braun, Melsungen für die Überlassung dieser Proben und des Lipofundin. 
$P$ - Verhältnis Lezithin : Kephalin $=10: 1,6$

$250 \mu \mathrm{g}$ Emulgator enthalten: $6,5 \mu \mathrm{g}$ Lezithin $-\mathrm{P}$ $1,01 \mu \mathrm{g}$ Kephalin $-\mathrm{P}$

\section{Zusammensetzung der Emulsion}

\section{Dïnnschichtchromatograpbie:}

Quantitative Phosphor-Bestimmung

1) Lezithin + Lysokephalin: $84,5 \%$

2) Kephalin : $6,8 \%$

3) Lysolezithin : $8,8 \%$

Verhältnis: $1: 2: 3=10: 0,8: 1$

Qualitativ nachzuweisen:

Glyzeride

Kephalin

Lezitbin

Lysokephalin, Lj'solezithin

Gesamt-Phosphor: $460 \mu \mathrm{g} \mathrm{P} / \mathrm{ml}=46,0 \%$

Phosphatidgehalt des Emulgators: $76,6 \%$

Gesamtfett (gravimetrisch): $100 \mathrm{ml}$,Lipofundin“ (10\% Baumwollsaatöl, 1,5\% Phosphatide) enthielten 13,22\% Gesamtfett.

\section{Ergebnisse}

Wie die in den Tabellen 3 und 4 dargestellten Ergebnisse zeigen, hält bei den Patienten mit einem erhöhten Nüchternwert für Gesamt-Esterfettsäuren ${ }^{1}$ ) als Ausdruck der bestehenden Triglyzeridtransportstörung die Infusionshyperlipämie länger an und erreicht absolut höhere Gipfelwerte als bei normolipämischen Personen. Die im einzelnen beobachteten Durchschnittswerte der Zunahme der Gesamtesterfettsäuren gegenüber dem Ausgangswert in der zweiten Stunde post infusionem betrugen: Gruppe $\mathrm{I}=+29 \%$; Gruppe $\mathrm{II}=+67 \%$ und Gruppe III $=+148 \%$. Besonders bemerkenswert ist das Verhalten der Fettwerte bei den Patienten der Gruppe III, die wegen ihrer Grundkrankheit über mehrere Tage jeweils 500-1000 ml der 10\%igen Fettemulsion erhalten hatten ${ }^{2}$ ), und bei denen die weitere intravenöse Er-

1) Auf die Berechnung der Triglyzeride wurde verzichtet.

2) Eine ausführliche Darstellung der Ergebnisse der Langzeittherapie mit Fettemulsionen siehe (15).

\section{Tab. 1}

Fettsäuremuster des Emulgators (Gaschromatogramm; Mittelwert von 3 Bestimmungen; Durchfluß $94 \mathrm{ml} / \mathrm{min}$. Helium, Temperatur $194^{\circ}$; Säule $200 \times 0,645 \mathrm{~cm}$, flüssige Phase: ButandiolsukzinatPolyester; Gerät: Perkin-Elmer 116 E) $v=$ verzweigte Monokarbonsäure.

\begin{tabular}{ccl}
\hline $\begin{array}{c}\text { Anzahl der } \\
\text { C-Atome }\end{array}$ & $\begin{array}{c}\text { Prozentualer } \\
\text { Anteil }\end{array}$ & Fettsäuren \\
\hline$<$ & & \\
\hline 8 & $1,8 \%$ & Kaprylsäure \\
10 & $5,5 \%$ & Kaprinsäure \\
13 & $0,2 \%$ & Tridekansäure \\
14 & $0,2 \%$ & Myristinsäure \\
15 & $0,1 \%$ & Pentadekansäure \\
16 & $0,06 \%$ & Palmitinsäure \\
& $12,5 \%$ & \\
$16=$ & & Palmitoleinsäure \\
17 & $0,2 \%$ & Heptadekansäure \\
$18 \nabla$ & $0,2 \%$ & ? (16= ?) \\
18 & $0,2 \%$ & Stearinsäure \\
$18=$ & $3,8 \%$ & Oleinsäure \\
$18={ }^{2}$ & $12,0 \%$ & Linolsäure \\
$18={ }^{3}$ & $52,3 \%$ & Linolensäure \\
20 & $10,1 \%$ & Arachidinsäure \\
22 & $0,4 \%$ & Behensäure \\
\hline
\end{tabular}

Tab. 2

Fettsäuremuster des Baumwollsaatöles (Gascbromatogramm; Mittelwert von 3 Bestimmungen; Versuchsbedingungen siehe Tab. 1).

\begin{tabular}{ccl}
\hline $\begin{array}{c}\text { Anzahl der } \\
\text { C-Atome }\end{array}$ & $\begin{array}{c}\text { Prozentualer } \\
\text { Anteil }\end{array}$ & Fettsäuren \\
\hline 8 & $0,05 \%$ & \\
8 & $0,04 \%$ & Kaprylsäure \\
10 & $0,04 \%$ & Kaprinsäure \\
12 & $0,1 \%$ & Laurinsäure \\
14 & $1,0 \%$ & Myristinsäure \\
15 & $0,06 \%$ & Pentadekansäure \\
$16 \mathrm{v}$ & $0,13 \%$ & $?$ \\
16 & $19,7 \%$ & Palmitinsäure \\
& & \\
17 & $0,4 \%$ & Heptadekansäure \\
$18 \mathrm{v}$ & $0,5 \%$ & ? \\
18 & $2,7 \%$ & Stearinsäure \\
$18=$ & $18,4 \%$ & Oleinsäure \\
$18=^{2}$ & $54,1 \%$ & Linolsäure \\
$18=^{3}$ & $1,0 \%$ & Linolensäure \\
20 & $0,8 \%$ & Arachidinsäure \\
\hline
\end{tabular}

Tab. 3

Mittelwerte der Gesamt-Esterfettsäurenkonzentration der drei Versuchsgruppen. Bei der statistischen Prüfung wurden die Gruppen II und III mit der Gruppe I verglichen. Die intravenöse Fettzufuhr wurde nach Abnahme des Nüchternwertes begonnen und war nach 3 Stunden beendet. Weitere Blutproben wurden nach der Infusion sowie 2 und 4 Stunden post infusionem entnommen.

\begin{tabular}{|c|c|c|c|c|c|c|c|c|c|}
\hline \multirow{2}{*}{$\begin{array}{l}\text { Unter- } \\
\text { suchungs- } \\
\text { gruppe }\end{array}$} & \multicolumn{9}{|c|}{ Bestimmung dex Gesamt-Esterfettsäuren im Serum. (Werte in mäq/100 ml) } \\
\hline & $\mathbf{n}$ & & $\begin{array}{l}\text { Ausgangswert } \\
\text { (vor Infusion) }\end{array}$ & & $\begin{array}{l}\text { i Infusionsende } \\
\text { lach } 3 \text { Stunden) }\end{array}$ & $2 \mathrm{Stu}$ & en post infusionem & 4 Stun & en post infusionem \\
\hline I & 10 & 1,02 & & 1,91 & & 1,33 & & 1,00 & \\
\hline II & 10 & 1,43 & $\begin{array}{l}\mathrm{S}_{D}= \pm 0,075 \\
\mathrm{P}=<0,001\end{array}$ & 3,04 & $\begin{array}{l}\mathrm{S}_{D}= \pm 0,248 \\
\mathrm{P}=<0,001\end{array}$ & 2,33 & $\begin{array}{l}\mathrm{S}_{D}= \pm 0,258 \\
\mathrm{P}=<0,001\end{array}$ & 1,90 & $\begin{array}{l}\mathrm{S}_{D}= \pm 0,106 \\
\mathrm{P}=<0,001\end{array}$ \\
\hline III & 6 & 1,79 & $\begin{array}{l}S_{D}= \pm 0,301 \\
P=<0,02\end{array}$ & 4,98 & $\begin{array}{l}\mathrm{S}_{D}= \pm 0,449 \\
\mathrm{P}=<0,001\end{array}$ & 4,08 & $\begin{array}{l}S_{D}= \pm 0,302 \\
P=<0,001\end{array}$ & 3,49 & $\begin{array}{l}S_{D}= \pm 0,396 \\
P=<0,001\end{array}$ \\
\hline
\end{tabular}


nährungstherapie wegen dieser Klärunfähigkeit ausgesetzt werden mußte. Damit stehen unsere Ergebnisse im Gegensatz zu denen der Voruntersucher $(6,7)$.

\section{Diskussion}

Zur Klärung dieser Diskrepanz muß zunächst darauf hingewiesen werden, da $B$ beide Arbeitsgruppen die Fettemulsion „Lipomul“ i. v. verwendeten, die $15 \%$ Baumwollsaatöl, 1,2\% Sojaphosphatide und 0,3\% Pluronic F $68^{1}$ ) enthält. Sowohl Mashrord (6) als auch BOUCHIER (7) bedienten sich zur Kontrolle der Lipämie jedoch der Trübungsmessung - - ein Verfahren, das
Koronarsklerose. Diese Erklärung gewinnt dadurch an Wahrscheinlichkeit, da $ß$ wir bei Untersuchungen über den Fetttransport nach intravenöser Fettzufuhr feststellten, daß pro Stunde nicht mehr als $15-20 \mathrm{~g}$ Fett aus dem Blut geklärt werden können. Selbst bei dieser Dosierung, stärker aber bei noch höherem Fettangebot, kommt es zu bemerkenswerten Veränderungen in den fetttransportierenden Eiweißfraktionen des Plasmas (Abb. 1). Wie wir zusammen mit BERG $(16,17)$ mittels Dünnschichtelektrophorese auf Stärke nachweisen konnten, findet sich post infusionem ein stark vermehrter Fettgehalt in allen Lipide und Lipoide transportierenden Fraktionen des Plasmas. Daß es sich dabei im wesent-
Abb. 1

Dünnschichtelektrophorese auf Stärke: Fett- und Eiweißfärbung auf dem $\mathrm{Ab}$ klatsch. Ein Vergleich vor und nach Ultrazentrifugieren zeigt, daß das zugeführte Fett in allen Globulinfraktionen transportiert wird (normalerweise in $\operatorname{det} \alpha_{.2}$ - und $\beta$-Fraktion; vgl. (17))

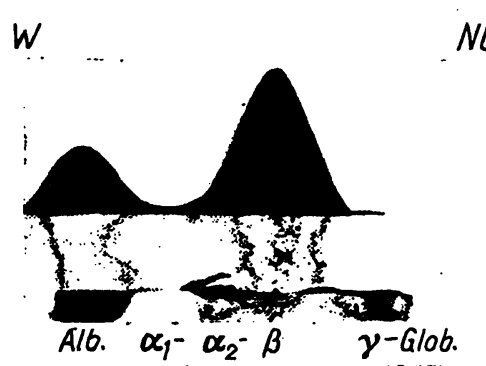

NÜCHTERN
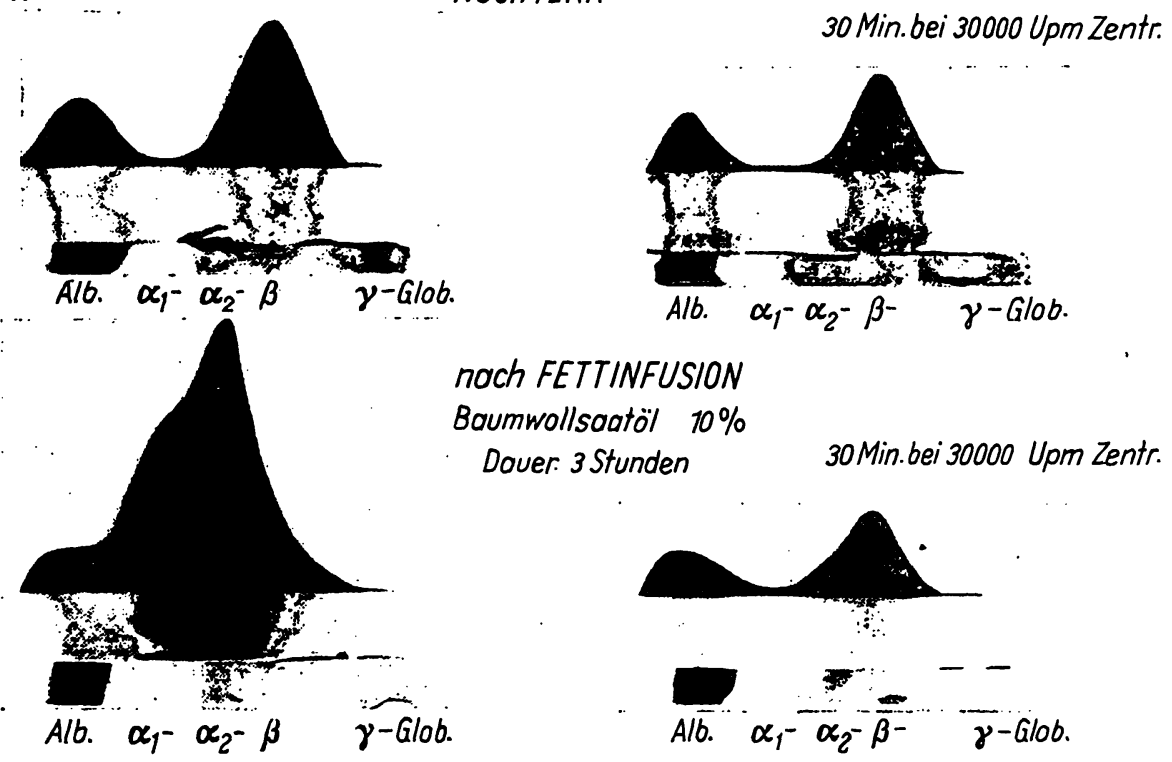

nach FETTINFUSION

Baumwollsaatöl $10 \%$

Dover. 3 Stunden

30Min.bei 30000 Upm Zentr.

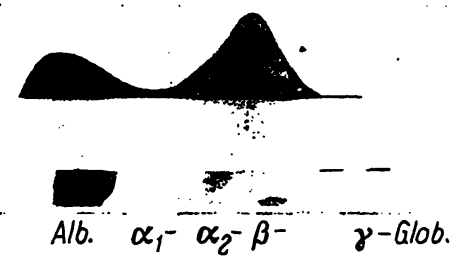

u. E. zur Untersuchung dieser Fragestellung nicht ausreicht. Außerdem war von diesen Autoren die Fettemulsion so schnell verabreicht worden $(12 \mathrm{~g}$ in 30 Minuten (6) bzw. $90 \mathrm{~g}$ in zwei Stunden (7), daß es zu einer ausgesprochenen Dyslipämie kommen mußte, bei der sich gefäßgesunde Patienten ebenso pathologisch verhielten wie Patienten mit einer Klärinsuffizienz bei

1) Pluronic F $68=$ Polymeres Polyoxyäthylenoxypropylen.

Tab. 4

Mittelwerte für Gesamtcholesterin und Phosphatide

\begin{tabular}{|c|c|c|c|c|}
\hline & $\begin{array}{l}\text { Vor der } \\
\text { Infusion }\end{array}$ & $\begin{array}{c}\text { Post } \\
\text { infusionem }\end{array}$ & $\begin{array}{l}2 \text { Stunden } \\
\text { p. i. }\end{array}$ & $\begin{array}{l}4 \text { Stunden } \\
\text { p. i. }\end{array}$ \\
\hline $\begin{array}{l}\text { Unter- } \\
\text { suchungs- } \\
\text { gruppe }\end{array}$ & \multicolumn{4}{|c|}{$\begin{array}{l}\text { Mittelwerte des Gesamtcholesteringehaltes } \\
\text { in } \mathrm{mg} \%\end{array}$} \\
\hline I & 165,2 & 182,5 & 181,8 & 165,3 \\
\hline II & 217,5 & 249,1 & 228,2 & 221,3 \\
\hline III & 216,2 & 248,0 & 242,0 & 199,3 \\
\hline & \multicolumn{4}{|c|}{ Mittelwerte des Phosphatidgehaltes in $\mathrm{mg} \%$} \\
\hline I & 251,8 & 340,3 & 251,8 & 214,3 \\
\hline II & 265,2 & 319,0 & 334,0 & 314,2 \\
\hline III & 270,0 & 347,0 & 369,5 & 327,0 \\
\hline
\end{tabular}

lichen, trotz unterschiedlicher Wanderungsgeschwindigkeit, um leichte Lipoproteide handelt, zeigt die Fettfärbung nach Ultrazentrifugierung. Dieses "Überlaufen“ läßt sich dadurch erklären, daß nach Sättigung des Transportvermögens der Proteine im $\beta$ - und $\alpha_{2}$-Globulinbereich auch schneller wandernde Lipoproteidfraktionen vikariierend eintreten, insbesondere auch solche Fraktionen, die normalerweise nur schwere Lipoproteide transportieren. Bereits nach Infusion von $10,0 \mathrm{~g}$ des Sojaphosphatid-Emulgators als 2\%ige Emulsion zeigt sich eine mäßige Zunahme des Gesamtfettes und anderer Fettinhaltsstoffe im Serum (Abb. 2), die auf eine Mobilisierung endogener Fette zurückgeführt werden muß. Demnach konkurrieren bei der intravenösen Fettzufuhr zwei Vorgänge miteinander: 1. die Mobilisierung endogenen Fettes und 2. der Abtransport der infudierten Fettemulsion. Bei zu schneller Infusion oder Verabreichung einer zu großen Fettmenge pro Zeiteinheit können sich diese Vorgänge für kurze Zeit die Waage halten, oder den normalen Fetttransport erheblich stören. Das Auftreten einer Retentionshyperlipämie nach mehrfacher nicht überdosierter intravenöser Fettzufuhr bei einigen Patienten deutet auf eine Erschöpfung des Fettklärmechanismus hin. Aus unseren bisherigen Untersuchungen ergibt sich kein 
Anhaltspunkt zur Erklärung der Ursache. Die Art dieser Hyperlipämie läßt sich auf Grund der Verteilung von Cholesterin und Triglyzeriden der essentiellen und symptomatischen Hyperlipämie (Hypertriglyzeridämie) zur Seite stellen.

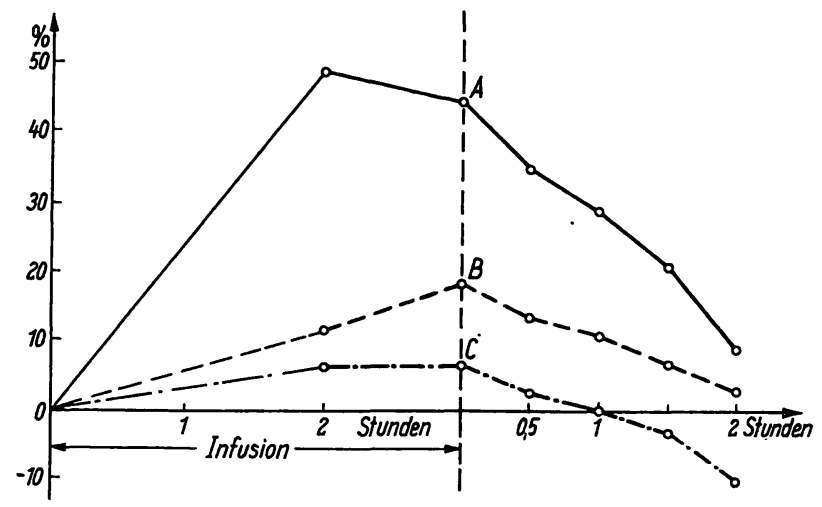

Abb. 2

Verhalten der Gesamt-Esterfettsäuren (A), der Phosphatide (B) und des Gesamt-Cholesterins (C) nach Verabreichung von $500 \mathrm{ml}$ einer $2 \%$ igen Sojaphosphatid-Emulgator-Emulsion in 3\% igem Sorbit in 3 Stunden
Aus den vorgelegten Untersuchungsergebnissen geht hervor, daß die intravenöse Fettbelastung wegen ihrer exakten Dosierbarkeit zur Untersuchung des Fetttransportes günstigete Voraussetzungen bietet als die orale Fettbelastung, der verschiedene Störfaktoren (Magenentleerung, Digestion und Resorption, Passagezeit) anhaften. Die zur intravenösen Fettbelastung notwenigen Triglyzerid- und Emulgatormengen lassen es jedoch möglich erscheinen, daß die Ergebnisse durch Mobilisierung endogenen Fettes (Depotfett oder Otganfett) beeinflußt werden, wie es aus den Arbeiten von Byers und FrIEDMANN $(18,19)$ und unseren eigenen Ergebnissen nach Phosphatidinfusion hervorgeht (Abb. 2). Zur quantitativen Abgrenzung dieses Faktors bedarf es weiterer Untersuchungen. Trotz dieses Umstandes kann die von uns angewandte Versuchsanordnung zusammen mit der Bestimmung der Gesamtesterfettsäuren (vgl. dazu $(10,11)$ ) als intravenöser Fettbelastungstest zur Bearbeitung spezieller Fragestellungen, wie z. B. der Prüfung von „kläraktiven“ Präparaten empfohlen werden. Eine radioaktive Emulsion mit labiler Markierung ( $\mathrm{J}^{131}$ ) würde eine weiter verbesserte Auswertung ermöglichen.

\section{Literatur}

1. Waldow A., J. E. Chapman und J. M. Evans, Amer. Heart J. 47, 568 (1954). - 2. Hauss, W. H. und E. BöHLE, Arch. klin. Med. 202, 579 (1955). - 3. BARRIT, D. W., Brit. Med. J. 2, 640 (1956). 4. Mitcheli, J. R. A. und B. Bronte-Stewart, Lancet 1, 167 (1959). - 5. Stanley, R. und S. J. Thannhauser, Trans. Assoc. Am. Phys. 40, 245 (1949). - 6. MAshrord M. L. und P. J. Nestel, Circulation Res. 9, 7 (1961). - 7. Bouchier, J. A. D. und B. Bronte-Stewart, Lancet 1,363 (1961). - 8. SchöN, H., Lancet II, 489 (1961). - 9.SснӧN, H., Verh. dtsch.Ges. inn. Med. 673 (1961). 10. SснӧN, H. und W. ZELLER, Münch. med. Wschr. im Druck. 11. Gey, F. und H. SснöN, Hoppe-Seyler's Z. physiol. Chem.
305, 149 (1956). - 12. Lohmann, K. und L. Jendrassik, Biochem. Z. 178, 419 (1926). - 13. Zlatkis, A., B. ZAK und A. J. Boyle, J. Lạb. Clin. Med. 50, 318 (1957). - 14. WAgner, H., Fette u. Seifen 63, 1119 (1961). - 15. Zeller, W., E. K. HAESE und H. SCHöN, Medizin und Ernährung, 3, 106 (1962). - 16. BERG, G. und H. ScнöN, Verh. dtsch. Ges. inn. Med. 883 (1961). - 17. SchöN, H., R. Flesch, W. Zeller und G. Berg, Med. Welt 28 , 1473 (1961). - 18. FriedmanN, M. und S. O. Byers, Amer. J. Physiol. 193, 435 (1957). - 19. Byers S. O., und M. Friedman; Proc. Soc. Exper. Biol. Med. 92, 459 (1956).

Dozent Dr. H. Schön Medizinische Universitäts-Klinik 852 Erlangen, Krankenhausstr. 12

Dic Wiedergabe von Gebraucbsnamen, Handelsnamen, Warenbezeichnunyen und dgl. in dieser Zeitscbrift berecbligt nicbl zu der Annabme, daß solcbe Namen obne weiteres von jedermann benülzt werden dürften. Vielmebr bandelt es sicb bäufg um gesetzlich gescbützte Warenzeicben, aucb wenn sie nicbl eigens als solcbe gekennzeicbnet sind.

Verlag Walter de Gruyter \& Co.,, vormals G. J. Göschen'sche Verlagshandlung - J. Guttentag, Verlagsbuchhandlung - Georg Reimer - Karl J. Trübner - Veit \& Comp., 1 Berlin 30, Genthiner Str. 13; 1963. - Printed in Germany. - Satz und Druck: Walter de Gruyter \& Co., 1 Berlin 30, Genthiner Str. 13. - Anzeigen: Merkur-Werbung, Dr. K. Jeserich KG, 7 Stuttgart 1, Postfach 740, Tel. 2463 58/59/50. - Für den Anzeigenteil verantwortlich: Willibald Plitzko. 